\title{
Advances in prenatal diagnostics
}

\author{
"I hope that the articles presented here will encourage engaging debate and \\ discussion regarding the status and future of biomarkers in the controversial field of \\ prenatal diagnostics."
}

\begin{abstract}
Keywords: biomarkers $\bullet$ fetal lung maturity $\bullet$ noninvasive $\bullet$ pre-eclampsia $\bullet$ prenatal diagnostics $\bullet$ preterm delivery $\bullet$ sequencing
\end{abstract}

Recent technical advances combined with concerns regarding the risks and limitations associated with traditional diagnostic approaches have led to dramatic changes in the often controversial field of prenatal diagnostics. Between 2006 and 2008, 261 women in the UK died as a direct or indirect result of pregnancy [1]. The overall maternal mortality rate in the UK for this period was 11.39 per 100,000 maternities, a decrease from previous years [1]. Despite improvements in maternal survival rates, there remain improvements to be made. Biomarkers to allow prenatal diagnosis of potential fatal conditions for both mother and baby will be key to future developments.

The themed articles in this issue of Biomarkers in Medicine aim to outline the discussion of the development and identification of prenatal diagnostic markers and techniques. Areas covered in the issue include pre-eclampsia, preterm birth, next-generation sequencing of fetal DNA and fetal lung maturity testing.

Between 2006 and 2008, pre-eclampsia was the second most common cause of maternal death in the UK, with little change in incidence from the earlier half of the decade [1]. Current estimates state that pre-eclampsia complicates between 4 and $7 \%$ of pregnancies worldwide. Difficulties in identifying those at risk and achieving a diagnosis may partially explain this lack of recent improvement, meaning the need for a reliable biomarker is paramount.

The first editorial article in this issue discusses the need for upstream biomarkers of pre-eclampsia, in order to relieve the current reliance on blood pressure and urinary protein markers [2]. Chappell focuses on two of the most promising markers currently being studied, placental growth factor and soluble fms-like tyrosine kinase. The article goes on to discuss the clinical and cost implications of both current and potential future approaches to diagnosis.

Also discussing potential markers of preeclampsia, the review in this issue by Griffin et al. focuses on clinical applications [3]. The authors give a thorough overview of current clinical practice and dilemmas. Potential novel biomarkers for first and second trimester detection are discussed, in addition to the potential for biomarker panels and combination of new biomarkers with other clinical parameters.

The leading cause of neonatal morbidity and mortality in high- and middle-income countries is preterm birth, commonly defined as birth prior to 37 weeks gestation [4]. Preterm birth is associated with high economic and emotional costs. It is hoped that identifying those at high risk through the use of biomarkers would allow the targeting of future research and interventions to those most likely to benefit.

The second review in this issue by Shennan and Foster discusses fetal fibronectin as a potential new biomarker of preterm birth [5]. Fetal fibronectin is a glycoprotein found in placental tissue, amniotic fluid and at the maternal-fetal interface. In this article, the authors review current data on the marker, in addition to discussing the potential for its integration into multimarker tests.

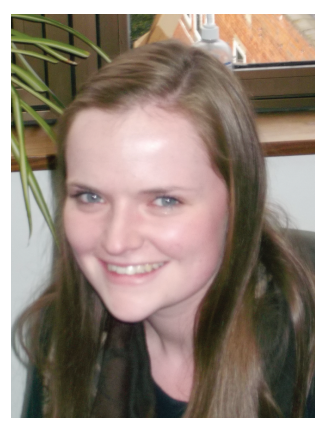

Hannah Wilson

Future Medicine Ltd, Unitec House, 2 Albert Place, London, N3 1QB, UK hannah.wilson@futuremedicine.com 
The effects of preterm birth can be lifelong, with many preterm infants going on to develop an aged phenotype, exhibiting adverse metabolic health through to adulthood [6]. In this issue, Turner et al. look at altered telomere attrition rates as a potential marker of this phenotype [6]. In their review, the authors conduct a thorough literature search on the topic and include comparative information on the various techniques available for telomere analysis.

Moving into the genomic era, Manegold-Brauer et al. review the implications of next-generation sequencing on prenatal diagnostics [7]. The ability to gain fetal genetic information from the mother's blood has already been clinically applied in tests for trisomy 21. In this review, the authors discuss current sequencing techniques, their prenatal applications, limitations and impact. In addition, the controversial ethical and social concerns surrounding the use of these developing techniques in prenatal diagnostics are addressed.

As certain technologies advance, it is arguably inevitable that others will fall by the wayside. This is addressed in the review by Gronowski et al., which asks whether the fetal lung maturity test, used to predict the risk of respiratory distress syndrome, is now

\section{References}

1 Centre for Maternal and Child Enquiries (CMACE). Saving mothers' lives: reviewing maternal deaths to make motherhood safer: 2006-08. The eighth report on confidential enquiries into maternal deaths in the United Kingdom. BJOG 118(Suppl. 1), 1-203 (2011).

2 Chappell LC, Bramham K, Shennan A. Short-term prediction of preeclampsia: how close are we? Biomarkers Med. 8(4), 455-458 (2014).

3 Griffin M, Shennan AH. Clinical applications of biomarkers in preeclampsia. Biomarkers Med. 8(4), 459-470 (2014).

4 Blencowe H, Cousens S, Oestergaard MZ. National, regional, and worldwide estimates of preterm birth rates in the year 2010 with time trends since 1990 for selected obsolete [8]. Respiratory distress syndrome remains a significant global cause of neonatal morbidity and mortality; however, the testing of lung surfactant in amniotic fluid to predict risk of the syndrome has been removed from most clinical recommendations. In this review, an overview of fetal lung maturity tests and their recent development is given, followed by a discussion of trends in test utilization and respiratory distress syndrome prevalence.

In summary, the articles highlighted from this issue of Biomarkers in Medicine give a broad view of research ongoing in various fields of prenatal diagnostics. I hope that the articles presented here will encourage engaging debate and discussion regarding the status and future of biomarkers in the controversial field of prenatal diagnostics.

\section{Financial \& competing interests disclosure}

$\mathrm{H}$ Wilson is an employee of Future Medicine Ltd. The author has no other relevant affiliations or financial involvement with any organization or entity with a financial interest in or financial conflict with the subject matter or materials discussed in the manuscript apart from those disclosed.

No writing assistance was utilized in the production of this manuscript.

countries: a systematic analysis and implications. Lancet 379(9832), 2162-2172 (2014).

5 Foster C, Shennan A. Fetal fibronectin as a biomarker of preterm labor: a review of the literature and advances in its clinical use. Biomarkers Med. 8(4), 471-484 (2014).

6 Turner KJ, Vasu V, Greenall J, Griffin DK. Telomere length analysis and preterm infant health: the importance of assay design in the search for novel biomarkers. Biomarkers Med. 8(4), 485-498 (2014).

7 Manegold-Brauer G, Hahn S, Lapaire O. What does next-generation sequencing mean for prenatal diagnosis? Biomarkers Med. 8(4), 499-508 (2014).

8 Yarbrough ML, Grenache DG, Gronowski AM. Fetal lung maturity testing: the end of an era. Biomarkers Med. 8(4), 509-515 (2014). 